\title{
Luteolin inhibited proliferation and induced apoptosis of prostate cancer cells through miR-30 I
}

This article was published in the following Dove Press journal:

OncoTargets and Therapy

26 May 2016

Number of times this article has been viewed

\author{
Kun $\operatorname{Han}^{1, *}$ \\ Wei Meng ${ }^{2, *}$ \\ Jian-jun Zhang' \\ Yan Zhou' \\ Ya-ling Wang' \\ Yang Su' \\ Shu-chen Lin' \\ Zhi-hua Gan' \\ Yong-ning Sun' \\ Da-liu Min' \\ 'Oncology Department, Shanghai \\ Jiao Tong University Affiliated Sixth \\ People's Hospital, Shanghai, ${ }^{2}$ Institute \\ of Genetic Engineering of Southern \\ Medical University, Guangzhou, \\ Guangdong, People's Republic \\ of China \\ *These authors contributed equally \\ to this work
}

\begin{abstract}
Luteolin is a falvonoid compound derived from Lonicera japonica Thunb. Numerous reports have demonstrated that luteolin has anticancer effects on many kinds of tumors. This study investigated the effects of luteolin on prostate cancer (PCa), assessing the PC3 and LNCaP cells. The cell viability and apoptosis were assessed by performing Cell Counting Kit- 8 assay and Annexin V-fluorescein isothiocyanate/propidium iodide double staining. Luteolin was found to inhibit androgen-sensitive and androgen-independent PCa cell lines' growth and induced apoptosis. To uncover the exact mechanisms and molecular targets, microRNA (miR) array analysis was performed. miR-301 was found to be markedly downregulated. Then, the expression of miR-301 was retrospectively analyzed in the primary PCa tissues by quantitative reverse transcription polymerase chain reaction and in situ hybridization methods. According to the quantitative reverse transcription polymerase chain reaction results of miR-301, the $54 \mathrm{PCa}$ patients were divided into two groups: high and low miR-301 groups. The division indicator is a relative expression $\geq 5$. Compared to the low-expression group, high miR-301 expression was associated with a significantly shorter overall survival $(P=0.029)$. The proapoptotic gene, $D E D D 2$, was predicted to be the direct target of miR-301. It was clarified in accordance with bioinformatics and luciferase activity analyses. The overexpression of miR-301 by plasmid decreased the luteolin effect. Taken together, these results suggest that luteolin inhibits PCa cell proliferation through miR-301, the poor predictive factor of PCa.
\end{abstract}

Keywords: luteolin, prostate cancer, miR-301, DEDD2, apoptosis

\section{Introduction}

Prostate cancer $(\mathrm{PCa})$ is the most common malignant tumor in old men. The general methodology of the Global Burden of Disease 2013 study indicated PCa as the leading cause for cancer incidence in men (1.4 million cases in a year). ${ }^{1}$ Androgen deprivation therapy is the initial treatment for PCa. Almost all these patients eventually develop progressive castration-resistant PCa (CRPC), despite an initial favorable response. ${ }^{2,3}$ Advanced PCa shows limited response to the existing systemic therapies. New generation of therapies for advanced PCa is still a cause for researchers' concern. In the past decades, a lot of research has been conducted to discover the natural compounds with potential antitumor activity. Flavonoids are naturally occurring polyphenolic metabolites distributed throughout the plant kingdom, and are found in substantial amounts in fruits, vegetables, grains, nuts, seeds, tea, and traditional medicinal herbs. Luteolin $\left(3^{\prime}, 4^{\prime}, 5^{\prime}, 7^{\prime}\right.$-tetrahydroxyflavone), one kind of flavonoid compounds, has a C6-C3-C6 structure and possesses two benzene rings, a third oxygen-containing ring, and a 2-3 carbon double bond. It possesses many beneficial properties, including antioxidant, ${ }^{4}$ anti-inflammatory, ${ }^{5}$ antibacterial, ${ }^{6}$ and anticancer. ${ }^{7-10}$ Herein, the antineoplastic activity of luteolin on PCa was studied and the molecular mechanisms of its aggressiveness

\footnotetext{
Correspondence: Yong-ning Sun;

Da-liu Min

Oncology Department of Shanghai Jiao

Tong University Affiliated Sixth People's

Hospital, Yishan Road 600, Xuhui

District, Shanghai 200233, People's

Republic of China

Tel +86 21 38297000 Ext 67766

Fax +862164701361

Email ynsun2002@I26.com;

mindaliu1969@I63.com
} 
have been indicated. Some reports proposed that luteolin is a new ribosomal S6 kinase inhibitor, ${ }^{11,12}$ while others clarified that luteolin has an antiangiogenic effect through inhibition of vascular endothelial growth factor. ${ }^{13,14}$ MicroRNAs (miRs), a new class of small RNAs, were found to be able to influence the tumor progression by modulating both mRNA stability and the ability of mRNA to be translated into protein. ${ }^{15,16}$ It was assumed that miR takes part in luteolin cytotoxicity. We focused on the mechanism of action of luteolin and directed our study toward miRNAs. We compared the miRs in the PCa cells with and without luteolin using miR array filtering. miR-301 is verified as the most stable and significant miR by quantitative reverse transcription polymerase chain reaction (qRT-PCR) method. We further clarified that miR-301 takes part in the effect of luteolin on PCa cell. Moreover, we found it to be associated with PCa progression.

\section{Materials and methods Materials}

Luteolin was purchased from Sigma-Aldrich Co. (St Louis, MO, USA; catalog number L9283-10MG, purity $\geq 98 \%$ ) and was dissolved in dimethyl sulfoxide (Sigma-Aldrich Co.; catalog number D2650, purity $\geq 99.7 \%$, grade Hybri-Max ${ }^{\mathrm{TM}}$ ) to stock concentrations of $100 \mathrm{mM}$ at $-20^{\circ} \mathrm{C}$. Cell Counting Kit-8 (CCK-8) was purchased from Beyotime Institute of Biotechnology (Beijing, People's Republic of China; catalog number C0037). MagMAX FFPE Total Nucleic Acid Isolation Kit (catalog number 4463365) was purchased from Ambion Co (Austin, TX, USA). Lipofectamin RNAmax (catalog number 13778500), TRIzol (catalog number 15596-018) reagent, and SuperScript ${ }^{\circledR}$ II Reverse Transcriptase (catalog number 18064014) reagent were purchased from Thermo Fisher Scientific (Waltham, MA, USA). SYBR Premix Ex Taq II (catalog number DRR041A) was purchased from TAKARA (Kusatsu, Japan). Annexin V-fluorescein isothiocyanate (FITC)/propidium iodide (PI) double-staining kit was purchased from Kaiji Bio-tech Co. (Nanjing, People's Republic of China; catalog number KGA1030-100). Mouse antihuman monoantibody against c-MET (catalog number 3127) was obtained from Cell Signaling Technology, Inc (Beverly, MA, USA).

\section{Cell culture}

The PC3 and LNCaP cells were purchased from the cell bank of Chinese Academy of Sciences (Shanghai, People's Republic of China) and maintained in Dulbecco's Modified Eagle's Medium (Thermo Fisher Scientific, Waltham, MA, USA) containing 10\% heat-inactivated fetal bovine serum albumin (Hyclone, Logan, UT, USA) in 95\% humidified incubator with $5 \% \mathrm{CO}_{2}$ at $37^{\circ} \mathrm{C}$ with penicillin-streptomycin $(10,000 \mathrm{U} / \mathrm{mL})$. The cultures were split every second day and diluted to a concentration of $2 \times 10^{5}$ cells $/ \mathrm{mL}$. The ethics committee of Sixth People's Hospital Shanghai, does not require ethics approval for human cell lines.

\section{Cell viability assay}

The cells were seeded into 96-well culture plates at a density of $1.5 \times 10^{4}$ cells/well in a $100 \mu \mathrm{L}$ medium and were treated with different concentrations $(15-60 \mu \mathrm{M})$ of luteolin for 24 , 48, and 72 hours (Figure 1). As for the rescue test (Figure 2), after transfecting miR-301-MIMIC for 24 hours, the cells were treated with $30 \mu \mathrm{M}$ (the representable value near to half maximal inhibitory concentration [IC50]) luteolin for 24 hours. Viable cells were evaluated using the CCK-8 assay, according to the manufacturer's instructions. Briefly, the CCK-8 solution $(50 \mu \mathrm{L} /$ well $)$ added to the cells in 24 -well plates was incubated at $37^{\circ} \mathrm{C}$ for 4 hours, and the optical density of each well was read at $490 \mathrm{~nm}$ using a microplate reader (ELX800; BioTek Instruments Inc., Winooski, VT, USA). The viability was assessed with the following equation:

$$
\underset{\text { viability }(\%)}{\text { Cell }}=\frac{\text { Absorbance of sample }- \text { Absorbance of blank }}{\text { Absorbance of control-Absorbance of blank }} \times 100 .
$$

\section{Cell apoptosis assay}

Apoptotic and dead cell counts were performed using FITClabeled Annexin V and PI staining (BD Biosciences, San Jose, CA, USA) by flow cytometry. The cells were collected and resuspended in binding buffer at a concentration of $3 \times 10^{6} / \mathrm{mL}$; then, $100 \mu \mathrm{L}$ of cell suspension was added to $5 \mu \mathrm{L}$ of Annexin V-FITC and $10 \mu \mathrm{L}$ of PI and mixed for 15 minutes in dark at room temperature. Next, $400 \mu \mathrm{L}$ of phosphate-buffered saline was added to the solution. A FACScan instrument (BD Biosciences) was used to count the cells $\left(1 \times 10^{3}\right)$ at an excitation wavelength of $490 \mathrm{~nm}$. The CellQuest software (BD Biosciences) was used for data collection and processing.

\section{Detection of differentially expressed miRNAs by miRNA microarray}

The LNCaP cells treated with or without luteolin at its IC50 for 24 hours were harvested and subsequently analyzed using miRNA microarray (Kangcheng Biotech Company, Shanghai, People's Republic of China). Briefly, total RNA was isolated from the cells using TRIzol reagent. The miRNA was separated from 30-50 mg of the total RNA, labeled with the miRCURY Hy3TM/Hy5TM Power Labeling Kit (Exiqon, 

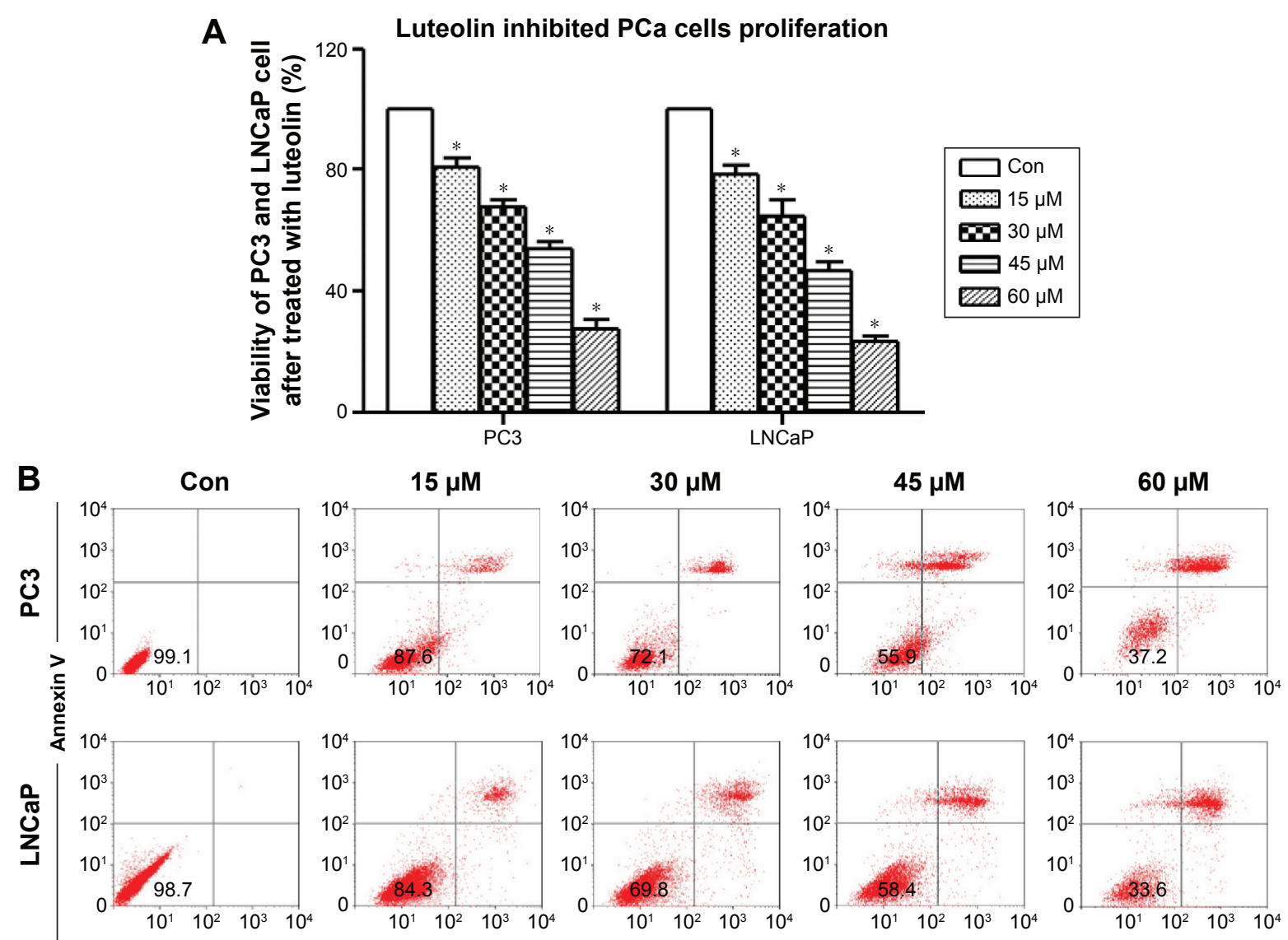

Figure I Luteolin regulates the cell proliferation, apoptosis, and chemosensitivity in PCa cells.

Notes: (A) After exposure to various concentrations (I5-60 $\mu \mathrm{M}$ ) of luteolin for 24 hours, viability of PC3 and LNCaP cells was determined using the CCK-8 assay. The viability ratio decreased in a dose-dependent manner ( $* \mathrm{P}<0.0 \mathrm{I}$ compared with the untreated control group). (B) PC3 and LNCaP cells were treated with I5-60 $\mu \mathrm{M}$ luteolin for 24 hours and stained with Annexin $\mathrm{V}$ and PI. The apoptosis ratio was detected by FCM. The numbers in the first quadrant were the living cell ratio.

Abbreviations: Con, control; CCK-8, Cell Counting Kit-8; PCa, prostate cancer; PI, propidium iodide; FCM, flow cytometry.

Vedbaek, Denmark), and hybridized to an miRCURY LNA Array (Exiqon, v11.0). Scanning was performed with an Axon GenePix 4000B microarray scanner (Axon Instruments Inc, Union City, CA, USA). GenePix Pro v6.0 was used to read the raw image intensity. Unsupervised hierarchical clustering was performed on the miRNA expression profile. Each miRNA present in the database was mapped to a precise location in the human genome using a Basic Local Alignment Search Tool (BLAST) search with the default parameters and the maps available from the National Center for Biotechnology Information Human Genome Resources (www.ncbi.nlm.nih.gov). In addition, to validate the present study data, the clones corresponding to each miRNA were identified and mapped to the human genome.

\section{Patients and specimens}

The present study is a retrospective analysis which includes 54 patients with primary PCa newly diagnosed from July 2005 to July 2010, who underwent consecutive therapy at the Shanghai Jiao Tong University Affiliated Sixth People's Hospital, People's Republic of China. This retrospective analysis was approved by the Research Ethics Committee of Shanghai Sixth People's Hospital. All patients voluntarily joined this study and signed informed consents. This study was designed to evaluate the 5-year survival rate. Ten pathological sections were collected for each patient to analyze the miR301 levels at the mRNA levels using the qRT-PCR method.

\section{Real-time qRT-PCR}

The MagMAX FFPE Total Nucleic Acid Isolation Kit was used to purify total miRNA from formalin-fixed, paraffinembedded tissue samples. The miR-301 and U6 primer was purchased from Jima Co. (Shanghai, People's Republic of China). Total RNA was extracted from PCa cells using the TRIzol reagent, according to the manufacturer's instructions. The complementary DNA was synthesized using the Murine Leukemia Virus Transcriptase Kit (Thermo Fisher Scientific). The quantitative analysis of DEDD2 mRNA level 
A Position 644-650 of DEDD2 3'-UTR 5'...GAGCUGAACAUAGACUUGCACUU...

hsa-miR-301 3'...CGAACUGUUAAUAGUAACGUGAC...
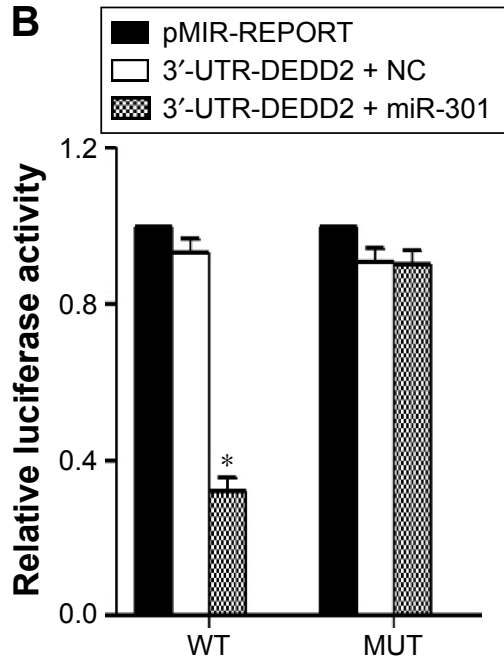
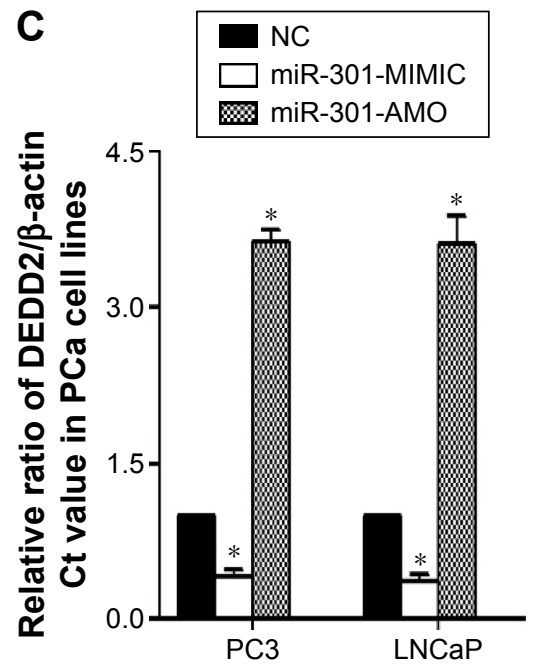

D

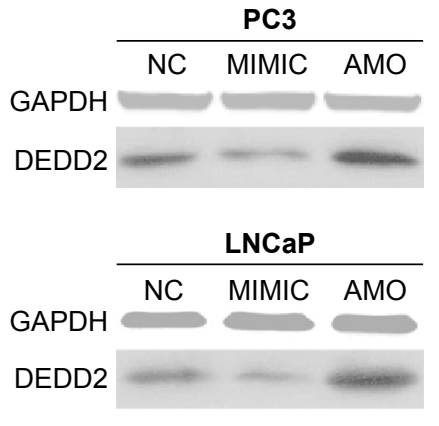

E

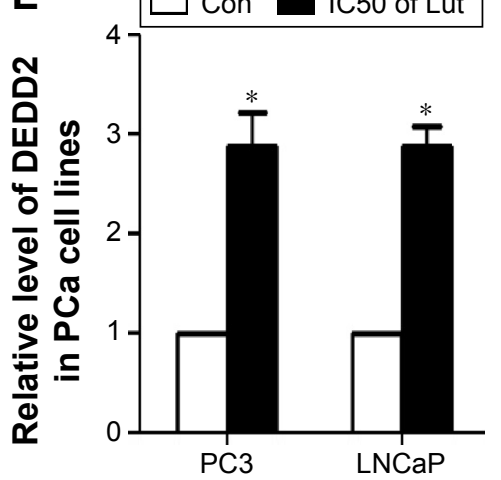

$\mathbf{F}$

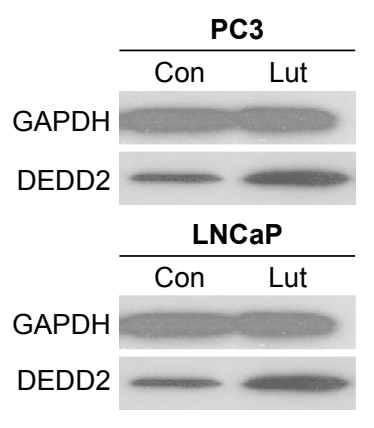

Figure 2 Functional screening of miR-30I target sites using luciferase reporter assay.

Notes: (A) The selection criteria of the miRNA targets were based on their common detection in the target prediction online databases (TargetScan5.I http://www.targetscan.org), as well as the full complementarity between the seed region of miR-30I and the 3'-UTR of DEDD2. (B) HEK-293T cells were cotransfected with miR-30I-MIMIC, psi-Check2, WT-psi-Check2, or MUT-psi-Check2 of DEDD2 gene. The luciferase activity levels were measured 48 hours after transfection. The results from at least three independent experiments are presented as the mean $\pm S E$. In this panel, the luciferase assay results show the regulation of $D E D D 2$ by miR-30I ( $* P<0.01$ compared with the control). (C) The DEDD2 mRNA expression levels in PC3 and LNCaP cells transfected with miR-30I-MIMIC or miR-30I-AMO were determined by qRT-PCR ( ${ }^{* P}<0.01$ compared with the control). (D) The DEDD2 protein expression levels in PC3 and LNCaP cells transfected with miR-30I-MIMIC or miR-30I-AMO were examined by Western blot analysis. (E) After exposure to IC50 luteolin, the mRNA levels of DEDD2 in both PC3 and LNCaP cells were found to be increased $>2.5$ times with qRT-PCR. (F) The protein levels of DEDD2 in PC3 and LNCaP cells had the same tendency toward mRNA.

Abbreviations: Con, control; GAPDH, glyceraldehyde 3-phosphate dehydrogenase; DEDD2, death effector domain containing 2; AMO, anti-miRNA oligonucleotide; Lut, luteolin; IC50, half maximal inhibitory concentration; miRNA, microRNA; MUT, mutate; NC, negative control; PCa, prostate cancer; pMIR-Report, untreated control; qRT-PCR, quantitative reverse transcription polymerase chain reaction; SE, standard error; UTR, untranslated region; WT, wild type.

was performed using qRT-PCR, and $\beta$-actin was used as an endogenous control. The sequences of the qRT-PCR primers were for DEDD2, forward: 5'-GTGTCTCCAGAACGCT-3' and reverse: 5'-AGAAGGCGTCCAGA-3'; and for $\beta$-actin, forward: 5'-TGAAGTGTGACGTGGACATC-3' and reverse: 5'-GGAGGAGCAATGATCTTGAT-3'. qRT-PCR was conducted using the Connect Real-Time PCR platform (Bio-Rad Laboratories, Hercules, CA, USA). The fold changes were calculated through the relative quantification using $2^{-\Delta \Delta \mathrm{Ct}}$. All the reactions were performed in a $20 \mu \mathrm{L}$ reaction volume in triplicate.

\section{In situ hybridization analysis}

$\mathrm{PCa}$ tissues for in situ hybridization (ISH) were fixed in $4 \%$ paraformaldehyde solution, dehydrated in a graded series of ethanol baths, and embedded in paraffin. Then, the tissue slides were deparaffinized and digested with proteinase $\mathrm{K}$ for 30 minutes. After that, the slides were prehybridized in a hybridization solution at $57^{\circ} \mathrm{C}$ for 2 hours. Tissues were hybridized overnight in the presence of $10 \mathrm{ng} 3^{\prime}-5^{\prime}$ biotinlabeled miR-301 (Exiqon) probes at $63^{\circ} \mathrm{C}$. Slides were washed twice stringently and an immunological reaction was carried out by using the rabbit antibody against biotin 
and alkaline phosphatase, according to the manufacturer's recommendation. Each side was assigned a score for intensity and staining positive pattern.

\section{Targeted in vitro luciferase reporter assay}

The psi-Check 2 plasmid (Promega Corporation, Fitchburg, WI, USA) was used for the expression of the miR-301 target DEDD2 3'-untranslated region (UTR). The wild-type (WT) Check2-DEDD2 construct contained two miR-301 response elements from the DEDD2 3'-UTR. In this study we mutated (MUT) both sites in Check2-DEDD2 construct which had two nucleotides and replaced $\mathrm{C}$ to $\mathrm{G}$ within the seed sequence. The sequences used to create the wild-type Check2-DEDD2 constructs were as follows: forward 5'-A ACTCGAGGACCCGCAGCAAATAGTTCA-3' (XhoI site shown in bold) and reverse 5'-AATGCGGCCGCC AATGTTCAGCGTAACCCAA-3' (NotI site shown in bold). The oligonucleotides were annealed and inserted into the psi-Check2 vector. Site-directed mutagenesis was performed using the QuikChange Kit (Agilent, Santa Clara, CA, USA). Mutations were introduced by replacing the predicted miR-301 binding site, AGCACUU, with AGGAGUU. The empty vector $(\overline{\mathrm{A}})$ was used as a negative control (NC). HEK-293T cells were transfected with 0.2 $\mu \mathrm{g}$ of the reporter plasmid and $0.01 \mu \mathrm{g}$ of the psi-Check2 control plasmid, and $200 \mathrm{nM}$ miR-301-MIMIC (miR-301 precursor) and the NC miRNA (Shanghai GenePharma Company, Shanghai, People's Republic of China) per well in 96-well plates. Following 24 hours of incubation, the cells were subjected to a luciferase reporter assay using the Luciferase Assay System (Promega Corporation). The Renilla luciferase values were normalized to firefly, and the ratio of Renilla/firefly values is presented. The experiments were performed independently in triplicate.

\section{Western blot analysis}

Protein lysates were resolved through 12\% sodium dodecyl sulfate-polyacrylamide gel electrophoresis and electrophoretically transferred to a polyvinylidene difluoride membrane (EMD Millipore, Billerica, MA, USA). Then, the membrane was probed with antibodies against human DEDD2 or glyceraldehyde 3-phosphate dehydrogenase, followed by probing with peroxidase-conjugated goat antimouse immunoglobulin G $(\mathrm{H}+\mathrm{L})$ (DingGuo, Shanghai, People's Republic of China) as a secondary antibody. The intensity of the protein fragments was visualized with an $\mathrm{X}$-ray image film-processing machine (Kodak, Rochester, NY, USA).

\section{Statistical analysis}

The data are expressed as mean \pm standard deviation of at least three independent experiments. One-way analysis of variance was used to test the differences between the mean value. All $P$-values were two sided, and a value of 0.05 was considered to be statistically significant. All statistical calculations were performed using Statistical Package for the Social Sciences software (version 18.0; SPSS Inc., Chicago, IL, USA).

\section{Results \\ Effect of luteolin on viability and apoptosis in PCa cells}

Luteolin has been reported to exhibit significant cytotoxicity against many kinds of cancer cells. ${ }^{17-19}$ In this study, the PC3 and $\mathrm{LNCaP}$ cells were treated with luteolin. The luteolin concentration range used in this experiment was $15-60 \mathrm{nM}$ for 24,48 , and 72 hours. The IC50 of each time period was calculated and a weighted average of these three results were taken, and the final IC50 of luteolin on PC3 and LNCaP cells was 31.44 and $32.05 \mu \mathrm{M}$, respectively. It resulted in a marked reduction of cell proliferation in a dose-dependent manner (Figure 1A). To understand the mechanism by which luteolin caused viability loss in PC3 and LNCaP cells, apoptosis experiments were carried out (Figure 1B).

\section{Luteolin downregulated the miR-30I expression}

In an attempt to search for the potential mechanisms by which luteolin induced apoptosis of PCa cells, the study identified the miRNAs modulated by luteolin in LNCaP cells for 24 hours using miRNA microarray (Figure 3A). miR-301 decreased by a factor of 5.39, which was found markedly downregulated after treatment of luteolin clarified by qRTPCR in both PC3 and LNCaP cells (Figure 3B).

\section{High expression of miR-30I, a poor predictive factor of $\mathrm{PCa}$ tissue}

Pathological section samples were collected from $54 \mathrm{PCa}$ patients in this study. The miR-301 expression was analyzed by qRT-PCR and ISH methods. The clinicopathological characteristics of $54 \mathrm{PCa}$ patients are shown in Table 1. The expression of miR-301 was related to distant metastasis, extracapsular extension, seminal vesicle invasion, stage, and Gleason score. No significant differences were noted in other clinical features such as age, prostate specific antigen (PSA), and surgical margin between individuals with high and low miR-301 expressions. Figure 4A reveals that the patients were divided into two groups: one was the high-expression group, 


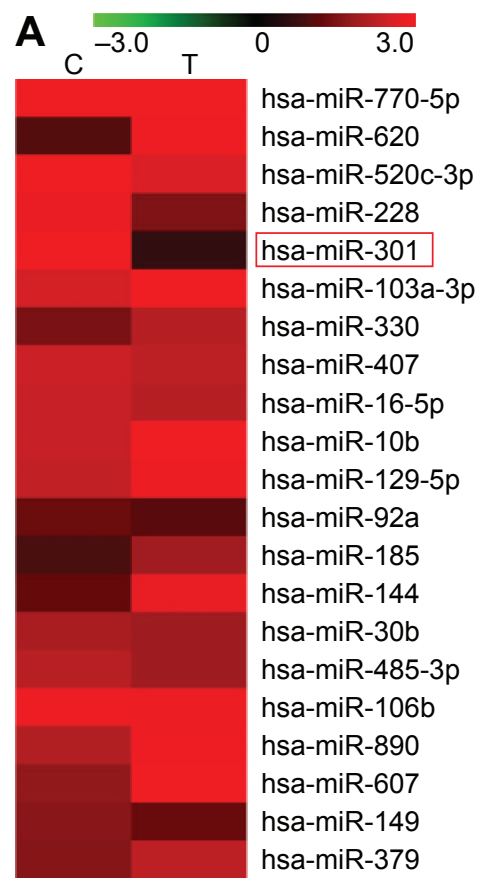

B

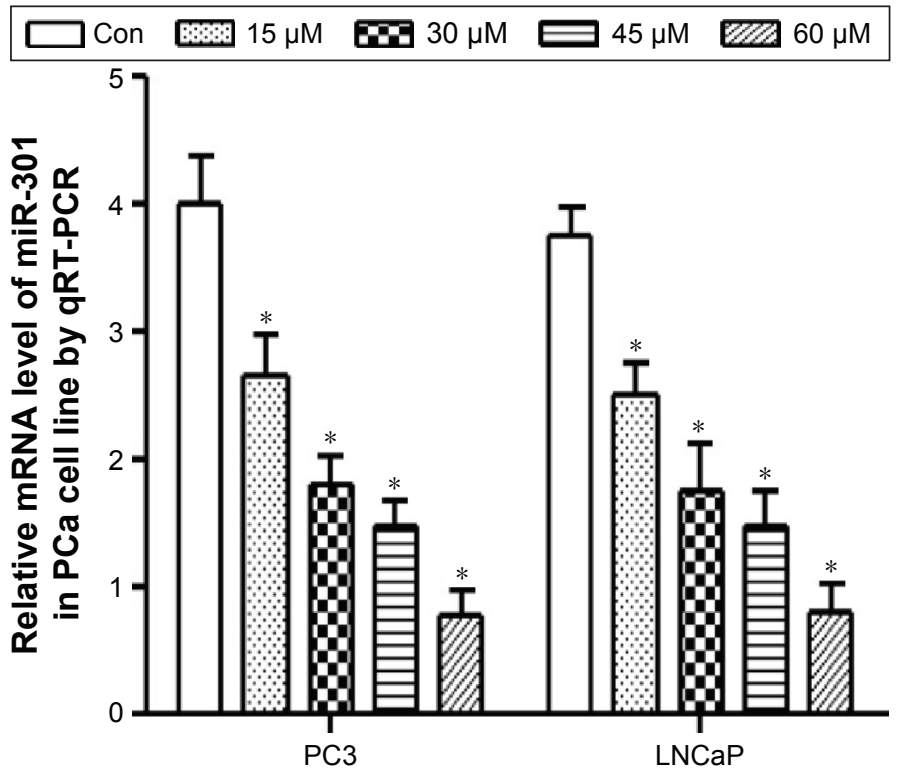

Figure 3 Luteolin downregulates miR-30I in LNCaP cells.

Notes: (A) Microarray analysis was used to compare the expression profiles of 703 miRNAs in LNCaP cells that were untreated (labeled as C) or treated (labeled as T) with luteolin. miR-30I, one of the most markedly downregulated miRs, is labeled with a red box. (B) As detected by the qRT-PCR method, the miR-30I levels dramatically decreased in a dose-dependent manner. $(* P<0.05)$

Abbreviations: Con, control; miRNA, microRNA; PCa, prostate cancer; qRT-PCR, quantitative reverse transcription polymerase chain reaction.

Table I Relationship between miR-30I expression and clinicopathological characteristics in PCa $(\mathrm{N}=54)$

\begin{tabular}{|c|c|c|c|}
\hline Patient characteristics & $\begin{array}{l}\text { Low miR- } \\
301 \text { group }\end{array}$ & $\begin{array}{l}\text { High miR- } \\
301 \text { group }\end{array}$ & $P$-value \\
\hline Age, years & & & 0.591 \\
\hline$<65$ & 24 & 8 & \\
\hline$\geq 65$ & 15 & 7 & \\
\hline Gleason score & & & 0.009 \\
\hline$\leq 8$ & 30 & 6 & \\
\hline$>8$ & 9 & 9 & \\
\hline Surgical margins & & & 0.148 \\
\hline $\mathrm{I} / \mathrm{II}$ & 33 & 10 & \\
\hline III/IV & 6 & 5 & \\
\hline PSA & & & 0.438 \\
\hline$<10 \mathrm{ng} / \mathrm{mL}$ & 12 & 3 & \\
\hline$\geq 10 \mathrm{ng} / \mathrm{mL}$ & 27 & 12 & \\
\hline Distant metastasis & & & 0.032 \\
\hline Negative & 30 & 7 & \\
\hline Positive & 9 & 8 & \\
\hline Extracapsular extension & & & 0.016 \\
\hline Negative & 33 & 8 & \\
\hline Positive & 6 & 7 & \\
\hline Stage & & & 0.017 \\
\hline Negative & 36 & 10 & \\
\hline Positive & 3 & 5 & \\
\hline Seminal vesicle invasion & & & 0.053 \\
\hline Negative & 33 & 9 & \\
\hline Positive & 6 & 6 & \\
\hline
\end{tabular}

Abbreviations: miR, microRNA; PCa, prostate cancer; PSA, prostate specific antigen. in which the relative level of miR-301 was $\geq 5$; and in the other group, the relative level of miR-301 was $<5$. KaplanMeier survival analysis showed that the high miR-301 group tended to have shorter overall survival (OS) (Figure 4B) than the low miR-301 group ( $P=0.029)$. Figure $4 \mathrm{C}$ shows the miR-301 level in the two groups using the ISH method.

\section{DEDD2, a direct target of miR-30 I}

The roles of oncogenic miRNAs are widely recognized in many cancers. There are some reports about miR-301. In this study, by TargetScan analysis, DEDD2 was predicted to be a potential miR-301 target site in 3'-UTR. The seed sequences for miR-301 in the $3^{\prime}$-UTR of DEDD2 are shown in Figure 2A. To determine whether miR-301 directly interacts with the 3'-UTR of DEDD2, the WT or MUT miR-301-DEDD2 response elements were cloned into the psiR-Check 2 plasmid downstream of the luciferase reporter, and HEK-293T cells were transfected with miR-301-MIMIC and the DEDD2 3 '-UTR vectors. As shown in Figure 2B, the luciferase reporter activity of WT, but not MUT Check2-DEDD2 inversely correlated with the miR-301 expression level. For the WT group, it was $\sim 20 \%-40 \%$ of the control group $(P<0.001)$. To know whether DEDD2 works as the downstream molecular target of miR-301, the DEDD2 level was analyzed by qRT-PCR (Figure 2C) and Western blot methods (Figure 2D). Figure 2C and $\mathrm{D}$ displays that after treated with miR-301-MIMIC and 
A

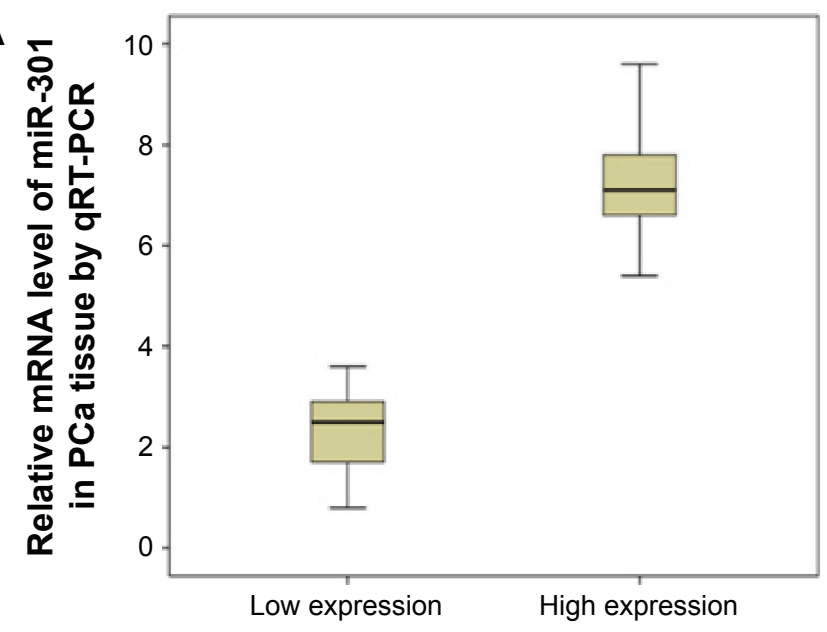

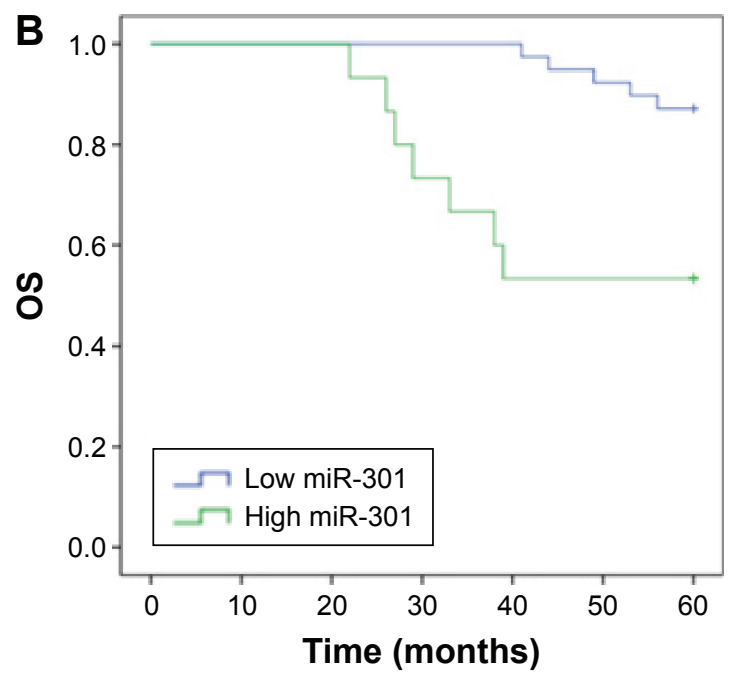

C
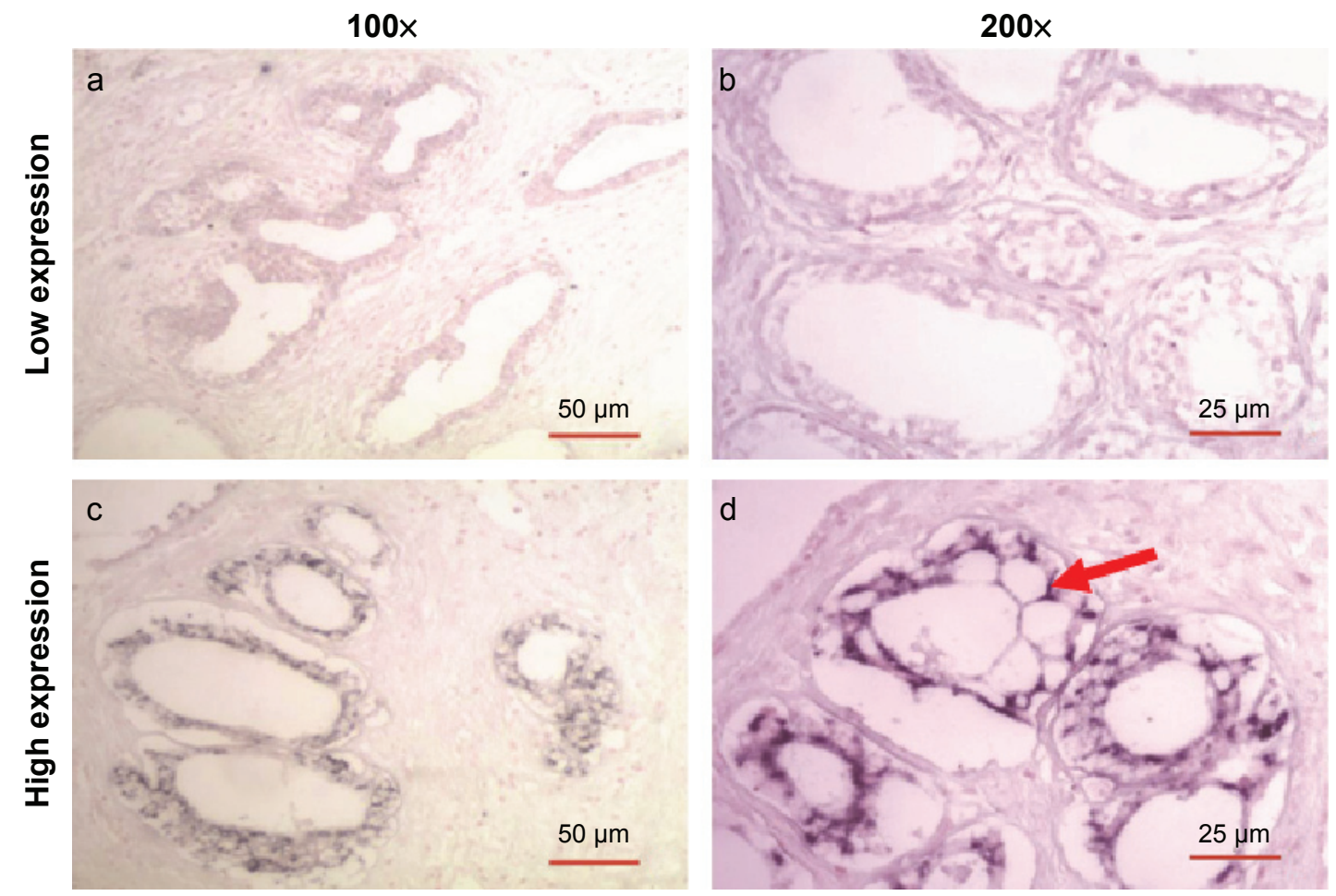

Figure 4 Correlation between the outcome and expression of miR-30I in PCa patients.

Notes: (A) Real-time PCR analysis of the miR-30I levels in 30 PCa tissues. The patients were divided into two groups. In the region marked "high expression", the miR-30 I relative level is $\geq 5$, and in the "low expression" region, it is $<5$. (B) Kaplan-Meier survival analysis of 30 PCa patients when high and low levels of miR-30I expression were compared. Significantly improved OS was observed in PCa patients whose tumors exhibited high miR-30I expression (green line) versus those whose tumors exhibited low miR-30I expression (blue line); the P-value is 0.029 . (C) miR-30I was detected in the PCa tissue by the ISH method, the darker particles in the plasma are positive. (a) and (b) are of the low-expression group; (c) and (d) are of the high-expression group. (a) and (c) are under I00×; (b) and (d) are under 200×. The red arrow points to the positive blue particle in the plasma.

Abbreviations: ISH, in situ hybridization; miRNA, microRNA; OS, overall survival; PCa, prostate cancer; PCR, polymerase chain reaction; qRT-PCR, quantitative reverse transcription polymerase chain reaction.

miR-301-AMO in PC3 and LNCaP cells for 24 hours, the mRNA and protein levels of DEDD2 changed with the change in miR-301 expression. After exposure to IC50 of luteolin, the mRNA level of DEDD2 in both PC3 and LNCaP cells were found to be increased $>2.5$ times by qRT-PCR (Figure 2E). The protein level of DEDD2 in PC3 and LNCaP cells had the same tendency toward mRNA (Figure 2F).

\section{Overexpression of miR-30I reversed the effects of luteolin on the PCa cells}

To examine the role of miR-301 in the generation of luteolin-induced functional responses, experiments were performed, in which PC3 and $\mathrm{LNCaP}$ cells were treated with the miR-301-MIMIC or control NC in the presence of luteolin (Figure 5A). The cell viability was determined 
by the CCK-8 assay (Figure 5B). In the group wherein luteolin was combined with miR-301-MIMIC, the miR-301 significantly countered luteolin's effect on cell viability, when compared with luteolin alone and the NC group. Similarly, when the induction of apoptosis was assessed, it was found that the apoptotic ratio of the miR-301-MIMIC group was significantly decreased (Figure 5C).

\section{Discussion}

PCa is an important age-related disease, being the most common cancer malignancy and the second leading cause of cancer mortality among men in Western countries. Androgen blockade therapy is believed to be the most effective and feasible treatment for PCa. ${ }^{20}$ However, each patient would progress to develop CRPC. The advanced PCa patients often poorly respond to chemotherapy and most of them are too old to tolerate chemotherapy, so they often have relatively poor prognosis. Recently, several new drugs have been approved that prolong the OS of CRPC, such as new-generation hormonal therapies, enzalutamide, and abiraterone acetate. Despite the demonstrated benefits of these agents, not all patients with CRPC are responsive to them; the appearance of acquired resistance in $<6$ months is almost universal. So, new drugs and clinical trials are needed to improve the outcome of PCa. Luteolin, a flavonoid found in $>300$ plant species (many of which are readily available in the human diet), has recently been shown to inhibit a variety of cancers, both in vitro and in vivo. ${ }^{21-23}$ In
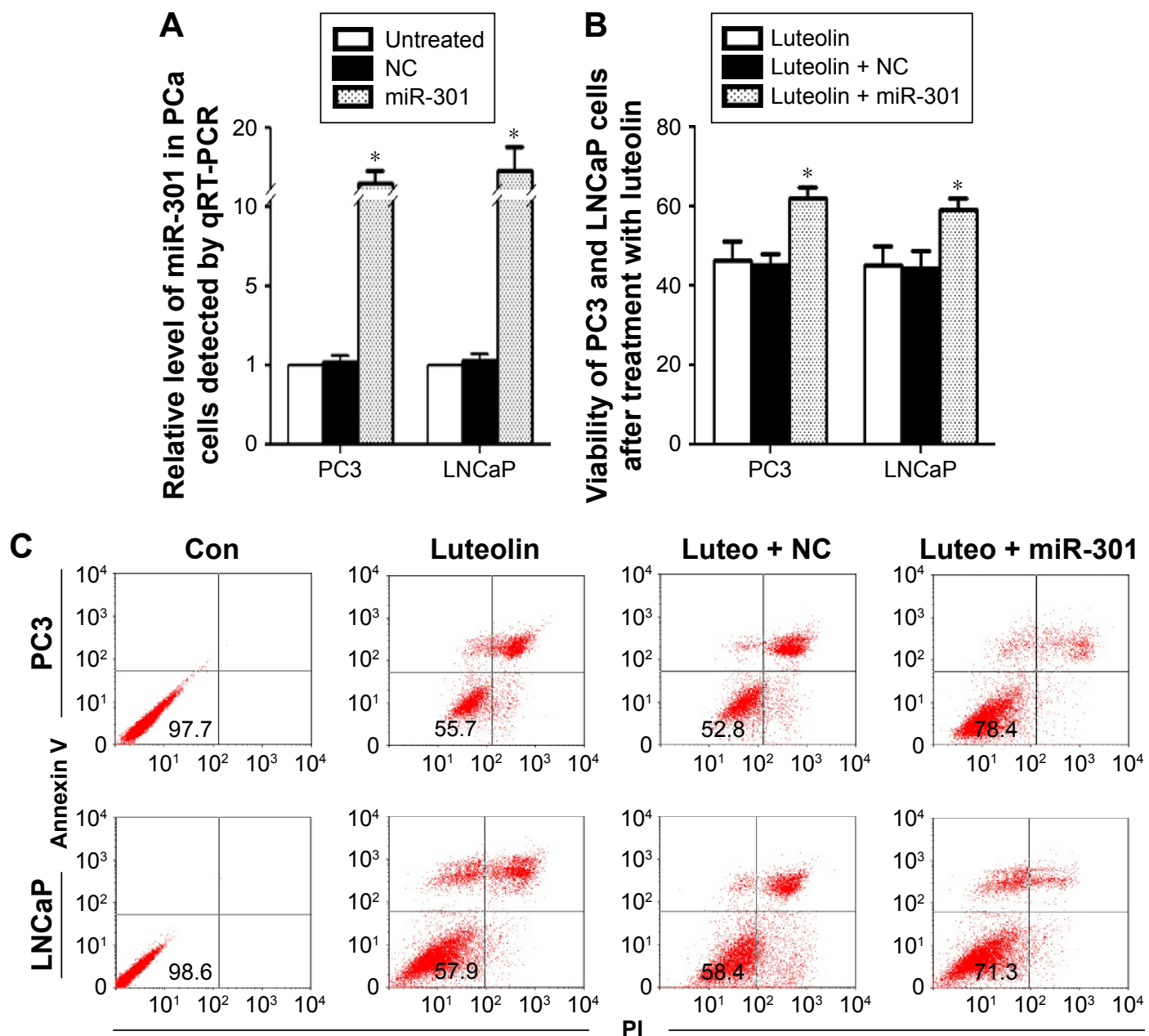

Figure 5 Reintroduction of miR-30I reverses the luteolin effect.

Notes: The PC3 and LNCaP cells were transfected with miR-30I-MIMIC for 24 hours and then treated with $30 \mu \mathrm{M}$ of luteolin. (A) After transfecting the cells with miR-30IMIMIC for 24 hours, the miR-30I level in both PCa cell lines increased more than ten times. (B) Cell viability was detected by CCK-8 method. miR-30I-MIMIC reversed the effects of luteolin $(30 \mu \mathrm{M})$ on PC 3 and LNCaP cells. The viability ratio was significantly higher than in both the control groups $(* P<0.01$, compared with the luteolin single group). (C) Changes in the apoptotic ratio of PC3 and LNCaP cells were detected by flow cytometry. The apoptotic ratio in the miR-30I-MIMIC group was markedly lesser than in the other two groups. The numbers in the first quadrant are the living cell ratios.

Abbreviations: Con, control; CCK-8, Cell Counting Kit-8; miRNA, microRNA; NC, negative control; PCa, prostate cancer; PI, propidium iodide; qRT-PCR, quantitative reverse transcription polymerase chain reaction. 
this study, we first found that luteolin inhibited proliferation and induced apoptosis in both $\mathrm{PC} 3$ and $\mathrm{LNCaP}$ cells with a dose-effect relationship. This paper reconfirmed that luteolin definitely has antitumor activity. Previous studies have indicated that luteolin exerts its antitumor actions by affecting numerous biochemical pathways critical for the regulation of cell survival, apoptosis, angiogenesis, and metastasis. ${ }^{24-26}$ Actually, its molecular mechanisms remain unclear. In this study, the focus was on the miRs, which participate in different biological processes, providing subtle combinational regulation of cellular pathways, often by regulating the components of signaling pathways. It is rarely reported which miR involved in them. miR-34 and miR-132 were upregulated by luteolin in neural tumor and gastric cancer. ${ }^{27-29}$ As we know, the miR expression is tissueand time-specific. What are the changes occurring in miRs in PCa cells exposed to luteolin? Actually, 16 kinds of miRs were upregulated or downregulated $>2.5$ times. As shown in Figure 3B, we found using qRT-PCR method that the change trend stability of miR-301 is stable and significant. So, we chose miR-301 as the focus. Sakurai et al reported that coadministration of luteolin and gefitinib to PC3 cells resulted in drastic downregulation of GAK protein expression through upregulation of miR-630. ${ }^{30}$ In this study, it was found that after exposure to luteolin, miR-301 was one of the markedly downregulated miRs. miR-301 is identified as an oncogenic gene and is upregulated in many cancers such as lung, breast, and pancreatic cancer. ${ }^{31-33}$ What is the role of miR-301 in PCa progression? In this study, the associations between the expression of miR-301 and the outcome of 54 cases of PCa were analyzed by both qRT-PCR and ISH methods. The expression of miR-301 was closely related to the stage, seminal vesicle invasion, and Gleason score. Kaplan-Meier survival analysis showed that the higher the miR-301 level was, the shorter OS the patients had (Figure 4B). Current clinical decisions on PCa management are based on parameters such as PSA, Gleason score, and tumor staging. Prostate biopsy and curative interventions are still disputed. The results of the present study indicated that miR-301 may be a potential effective predictor. miRs not only work as valuable prognostic biomarkers, but also emerged as gene regulators. The study predicted DEDD2, a proapoptotic factor, as a direct target of miR-301. Apoptosis is an important process to maintain cellular homeostasis, which is regulated and executed by specialized proteins. One of these domains is the death effector domain (DED). It carries protein-protein interaction effect and has predominantly been found in components of the death-inducing signaling complex. The main players in death-inducing signaling complex formation employ tandem DEDs for regulating signaling complex formation. The following are the seven DED-containing proteins that exist in human beings: FADD, c-FLIP, caspase-8, caspase-10, DEDD, DEDD2, and PEA-15. Deregulated apoptosis is a main phenomenon in cancer. Alcivar et $\mathrm{al}^{34}$ reported that DEDD2 targeted the nucleolus and was bound to two tandem DED-containing caspases, caspase- 8 and caspase- 10 . It may be an important mediator for death receptors. In this study, it was first clarified that DEDD2 is the direct target of miR-301. The level of DEDD2 changed with miR-301 on both mRNA and protein. In order to verify that DEDD2 plays an important role in luteolin's effects on PCa cells, the mRNA and protein levels of DEDD2 in PC3 and LNCaP cells with or without IC50 luteolin were detected by qRT-PCR and Western blot method (Figure 2E and F). The results indicated both mRNA and protein levels of DEDD2 were increased more than two times. The study next explored whether miR-301 is the key factor of luteolin's effect on PCa proliferation and apoptosis. The miR-301-MIMIC was transfected in PC3 and LNCaP cell lines for 24 hours and then treated with $30 \mathrm{nM}$ luteolin for 24 hours. The viability and apoptosis analysis supported that miR-301 plays an important role in luteolin-modulated cell proliferation and apoptosis of PCa cells.

\section{Conclusion}

The pooled analysis of this study demonstrated that luteolin inhibited PCa cell proliferation and induced apoptosis through downregulation of miR-301 by triggering DEDD2 expression. The miR-301 expression was significantly close to the outcome of PCa patients.

\section{Disclosure}

The authors report no conflicts of interest in this work.

\section{References}

1. Global Burden of Disease Cancer Collaboration, Fitzmaurice C, Dicker D, Pain A, et al. The global burden of cancer 2013. JAMA Oncol. 2015;1(4):505-527.

2. Sakamoto S, Ichikawa T. [Current status of castration resistant prostate cancer basic research]. Nihon Rinsho. 2014;72(12):2097-2102.

3. Mostaghel EA, Lin DW. Practical guide to the use of abiraterone in castration resistant prostate cancer. Can J Urol. 2014;21(2 Suppl 1): 57-63.

4. Plioukas M, Gabrieli C, Lazari D, Kokkalou E. Phytochemical analysis with the antioxidant and aldose reductase inhibitory capacities of Tephrosia humilis aerial parts' extracts. Nat Prod Res. 2015;24:1-7.

5. Nepali S, Son JS, Poudel B, Lee JH, Lee YM, Kim DK. Luteolin is a bioflavonoid that attenuates adipocyte-derived inflammatory responses via suppression of nuclear factor- $\mathrm{\kappa B} /$ mitogen-activated protein kinases pathway. Pharmacogn Mag. 2015;11(43):627-635. 
6. Lv PC, Li HQ, Xue JY, Shi L, Zhu HL. Synthesis and biological evaluation of novel luteolin derivatives as antibacterial agents. Eur J Med Chem. 2009;44(2):908-914.

7. Zheng $\mathrm{CH}$, Zhang M, Chen $\mathrm{H}$, et al. Luteolin from Flos Chrysanthemi and its derivatives: new small molecule Bcl-2 protein inhibitors. Bioorg Med Chem Lett. 2014;24(19):4672-4677.

8. Yang MY, Wang CJ, Chen NF, Ho WH, Lu FJ, Tseng TH. Luteolin enhances paclitaxel-induced apoptosis in human breast cancer MDA-MB-231 cells by blocking STAT3. Chem Biol Interact. 2014; 213:60-68.

9. Hwang JT, Park OJ, Lee YK, et al. Anti-tumor effect of luteolin is accompanied by AMP-activated protein kinase and nuclear factor- $\mathrm{KB}$ modulation in HepG2 hepatocarcinoma cells. Int J Mol Med. 2011; 28(1):25-31.

10. Amin AR, Wang D, Zhang H, et al. Enhanced anti-tumor activity by the combination of the natural compounds (-)-epigallocatechin3-gallate and luteolin: potential role of p53.J Biol Chem. 2010;285(45): 34557-34565.

11. Reipas KM, Law JH, Couto N, et al. Luteolin is a novel p90 ribosomal S6 kinase (RSK) inhibitor that suppresses Notch4 signaling by blocking the activation of Y-box binding protein-1 (YB-1). Oncotarget. 2013; 4(2):329-345.

12. Kim JE, Son JE, Jang YJ, et al. Luteolin, a novel natural inhibitor of tumor progression locus 2 serine/threonine kinase, inhibits tumor necrosis factor-alpha-induced cyclooxygenase-2 expression in JB6 mouse epidermis cells. J Pharmacol Exp Ther. 2011;338(3):1013-1022.

13. Lee EJ, Oh SY, Sung MK. Luteolin exerts anti-tumor activity through the suppression of epidermal growth factor receptor-mediated pathway in MDA-MB-231 ER-negative breast cancer cells. Food Chem Toxicol. 2012;50(11):4136-4143.

14. Ding H, Li D, Zhang Y, et al. Luteolin inhibits smooth muscle cell migration and proliferation by attenuating the production of Nox4, p-Akt and VEGF in endothelial cells. Curr Pharm Biotechnol. 2014; 14(12):1009-1015.

15. Hong M, Wang N, Tan HY, Tsao SW, Feng Y. MicroRNAs and Chinese medicinal herbs: new possibilities in cancer therapy. Cancers (Basel). 2015;7(3):1643-1657.

16. Goto Y, Kurozumi A, Enokida H, Ichikawa T, Seki N. Functional significance of aberrantly expressed microRNAs in prostate cancer. Int J Urol. 2015;22(3):242-252.

17. Liu X, Ye F, Wu J, How B, Li W, Zhang DY. Signaling proteins and pathways affected by flavonoids in leukemia cells. Nutr Cancer. 2015; 67(2):238-249.

18. Wu PS, Yen JH, Kou MC, Wu MJ. Luteolin and apigenin attenuate 4-hydroxy-2-nonenal-mediated cell death through modulation of UPR, Nrf2-ARE and MAPK pathways in PC12 cells. PLoS One. 2015;10(6): e0130599.
19. Sato Y, Sasaki N, Saito M, Endo N, Kugawa F, Ueno A. Luteolin attenuates doxorubicin-induced cytotoxicity to MCF-7 human breast cancers. Biol Pharm Bull. 2015;38(5):703-709.

20. Küronya Z, Bíró K, Géczi L, Németh $H$. Treatment strategies for advanced prostate cancer. Magy Onkol. 2015;59(3):229-240.

21. Ma L, Peng H, Li K, et al. Luteolin exerts an anticancer effect on NCIH460 human non-small cell lung cancer cells through the induction of Sirt1-mediated apoptosis. Mol Med Rep. 2015;12(3):4196-4202.

22. Fernando W, Rupasinghe HP, Hoskin DW. Regulation of hypoxiainducible factor- $1 \alpha$ and vascular endothelial growth factor signaling by plant flavonoids. Mini Rev Med Chem. 2015;15(6):479-489.

23. Lu J, Li G, He K, et al. Luteolin exerts a marked antitumor effect in cMet-overexpressing patient-derived tumor xenograft models of gastric cancer. J Transl Med. 2015;13:42.

24. Chen CT, Kim H, Liska D, Gao S, Christensen JG, Weiser MR. MET activation mediates resistance to lapatinib inhibition of HER2-amplified gastric cancer cells. Mol Cancer Ther. 2012;11(3):660-669.

25. Kim YS, Kim SH, Shin J, et al. Luteolin suppresses cancer cell proliferation by targeting vaccinia-related kinase 1. PLoS One. 2014;9(10): e109655.

26. Hong Z, Cao X, Li N, et al. Luteolin is effective in the non-small cell lung cancer model with L858R/T790M EGF receptor mutation anderlotinib resistance. Br J Pharmacol. 2014;171(11):2842-2853.

27. Chen PY, Wu MJ, Chang HY, Tai MH, Ho CT, Yen JH. Up-regulation of miR-34a expression in response to the luteolin-induced neurite outgrowth of PC12 cells. J Agric Food Chem. 2015;63(16):4148-4159.

28. Wu H, Huang M, Liu Y, Shu Y, Liu P. Luteolin induces apoptosis by up-regulating miR-34a in human gastric cancer cells. Technol Cancer Res Treat. 2014;14(6):747-755.

29. LinLF, Chiu SP, WuMJ,ChenPY, YenJH.LuteolininducesmicroRNA-132 expression and modulates neurite outgrowth in PC12 cells. PLoS One. 2012;7(8):e43304.

30. Sakurai MA, Ozaki Y, Okuzaki D, et al. Gefitinib and luteolin cause growth arrest of human prostate cancer PC-3 cells via inhibition of cyclin G-associated kinase and induction of miR-630. PLoS One. 2014; 9(6):e100124.

31. Nadal E, Truini A, Nakata A, et al. A novel serum 4-microRNA signature for lung cancer detection. Sci Rep. 2015;5:12464.

32. Funamizu N, Lacy CR, Parpart ST, Takai A, Hiyoshi Y, Yanaga K. MicroRNA-301b promotes cell invasiveness through targeting TP63 in pancreatic carcinoma cells. Int J Oncol. 2014;44(3):725-734.

33. Shi W, Gerster K, Alajez NM, et al. MicroRNA-301 mediates proliferation and invasion in human breast cancer. Cancer Res. 2011;71(8): 2926-2937.

34. Alcivar A, Hu S, Tang J, Yang X. DEDD and DEDD2 associate with caspase-8/10 and signal cell death. Oncogene. 2003;22(2):291-297.
OncoTargets and Therapy

\section{Publish your work in this journal}

OncoTargets and Therapy is an international, peer-reviewed, open access journal focusing on the pathological basis of all cancers, potential targets for therapy and treatment protocols employed to improve the management of cancer patients. The journal also focuses on the impact of management programs and new therapeutic agents and protocols on
Dovepress

patient perspectives such as quality of life, adherence and satisfaction. The manuscript management system is completely online and includes a very quick and fair peer-review system, which is all easy to use. Visit http://www.dovepress.com/testimonials.php to read real quotes from published authors. 